INT. J. GEOGRAPHICAL INFORMATION SCIENCE, 1998, VOL. 12, NO. 1, 47-61

Research Article

\title{
Accuracy of local topographic variables derived from digital elevation models
}

\author{
IGOR V. FLORINSKY \\ Institute of Mathematical Problems of Biology, Russian Academy of Sciences, \\ Pushchino, Moscow Region, 142292 Russia \\ email: flor@impb.serpukhov.su
}

(Received 25 February 1997; accepted 2 June 1997)

\begin{abstract}
We study the accuracy of data on some local topographic attributes derived from digital elevation models (DEMs). First, we carry out a test for the precision of four methods for calculation of partial derivatives of elevations. We find that the Evans method is the most precision algorithm of this kind. Second, we produce formulae for root mean square errors of four local topographic variables (gradient, aspect, horizontal and vertical landsurface curvatures), provided that these variables are evaluated with the Evans method. Third, we demonstrate that mapping is the most convenient and pictorial way for the practical implementation of the formulae derived. A DEM of a part of the Kursk Region (Russia) is used as an example. We find that high errors of data on local topographic variables are typical for flat areas. Results of the study can be used to improve landscape investigations with digital terrain models.
\end{abstract}

\section{Introduction}

Digital terrain models (DTMs) can be defined as digital representations of variables relating to a topographic surface, namely: digital elevation models (DEMs), digital models of gradient $(G)$, aspect $(A)$, horizontal $\left(k_{h}\right)$ and vertical $\left(k_{v}\right)$ landsurface curvatures as well as other topographic attributes (Miller and Leflamme 1958, Doyle 1978, Burrough 1986, Felicisimo 1994 a, Shary 1995). DTMs are extensively used in landscape investigations (Moore et al. 1991, Shary et al. 1991, Florinsky 1995). Application of local topographic variables, such as $G, A, k_{h}$ and $k_{v}$ has attracted considerable interest. This is due to the fact that these attributes are connected with processes of lateral migration and accumulation of water and other substances by gravity along the landsurface and in soil (Moore et al. 1991, Shary et al. 1991).

$G$ is the angle between a tangent and a horizontal plane in a given point of the landsurface. $A$ is the angle clockwise from north to a projection of a normal vector to a horizontal plane through a given point of the landsurface. $k_{v}$ is the curvature of a normal section of the landsurface compared to a plane including a gravity acceleration vector at a given point on the landsurface. $k_{h}$ is the curvature of a normal section of the landsurface; this section is orthogonal to the section with $k_{v}$ (Evans 1980, Shary 1991).

Local topographic variables can be derived from elevation $(z)$ values in a small neighbourhood of each point of the landsurface. $z$ is given by $z=f(x, y)$ where $x$ and $y$ are plan Cartesian coordinates, and $G, A, k_{h}$ and $k_{v}$ are functions $(\S 4)$ of the 
following partial derivatives (Shary 1991):

$r=\frac{\delta^{2} z}{\delta x^{2}}, t=\frac{\delta^{2} z}{\delta y^{2}}, s=\frac{\delta^{2} z}{\delta x \delta y}, p=\frac{\delta_{z}}{\delta x}$ and $q=\frac{\delta_{z}}{\delta y} . r, t, s, p$ and $q$

can be calculated with regular (square-gridded) DEMs by various methods (Sharpnack and Akin 1969, Evans 1980, Horn 1981, Papo and Gelbman 1984, Ritter 1987, Zevenbergen and Thorne 1987, Skidmore 1989, Moore et al. 1993, Shary 1995). These methods $(\$ 2)$ are based on approximation of differential operators by finite differences (Ames 1977).

It is obvious that errors of DTMs can adversely affect the accuracy and impartiality of investigation and modelling of natural processes. So, considerable study has focussed on aspects of DTM accuracy. For example, Carter (1988) discussed causes for errors in DEMs compiled by different methods. Researchers developed algorithms for detection of errors in DEMs (Frederiksen 1981, Hannah 1981, Brown and Bara 1994, Felicisimo 1994 a, 1994 b), estimation of these errors (Ackermann 1978, Felicisimo 1994 a, Li 1994), their visualization (Kraus 1994, Hunter and Goodchild 1995) and correction (Hannah 1981, Brown and Bara 1994, Felicisimo 1994a). Kumler (1994) performed a detailed comparison of the accuracy of eight types of DEMs for 25 terrain types. He demonstrated that the highest accuracy is specific to regular DEMs produced by linear interpolation of $z$ values from the strings of digitized contours.

The accuracy of $G, A$ and $k_{v}$ calculation is less well understood, while no consideration has been given to the accuracy of $k_{h}$ computation. Basically, the accuracy of digital models and maps of $G, A$ and $k_{v}$ was studied by a comparison of calculated and 'reference' values of these variables. For 'reference' data researchers used hand measurements of $G$ and $A$ from topographic maps (Evans 1980, Skidmore 1989), field measurements of $G, A$ and $k_{v}$ (Bolstad and Stowe 1994, Giles and Franklin 1996), $G$ and $A$ derived from 'reference' DEMs of actual surfaces (Chang and Tsai 1991) and imaginary ones (Carter 1992, Felicisimo 1995, Hodgson 1995). However, there is no reason to suppose that these 'reference' data, measurements and computations are correct. There are grounds to think that the accuracy of data on $G, A, k_{h}$ and $k_{v}$ cannot be determined by a comparison of calculated and 'reference' values (Shary, personal communication 1991). Really, a measurement accuracy can be defined as a difference between a measured value and an actual value of a variable (e.g., Gaidaev and Bolshakov 1969). However, the actual landsurface is not mathematically smooth. So, it cannot have derivatives and hence $G, A, k_{h}$ and $k_{v}$. These variables are abstract ones, and arise only during measurements (Shary 1991). As there are no actual values of $G, A, k_{h}$ and $k_{v}$, the accuracy of these data cannot be determined by a comparison of calculated and 'reference' values.

Moreover, this strategy can lead to some artefacts, subjective and conflicting conclusions. For instance, it was found that errors of $A$ calculation are typical for flat areas (Chang and Tsai 1991, Carter 1992), while errors of $G$ computation are predominantly positioned on steep slopes (Chang and Tsai 1991, Sasowsky et al. 1992, Bolstad and Stowe 1994). However, Carter (1992) emerged that errors in $G$ and $A$ become large in flat areas. At the same time, Davis and Dozier (1990) found that $G$ and $A$ errors concentrate within zones of rapid change in slope and exposure (e.g., ridges and ravines). Evans (1980) pointed out that the accuracy of $G, A, k_{h}$ and $k_{v}$ maps depends on the matrix step or cell size $(w)$ of a DEM. For example, small 
steep zones can transform to broad areas marked by medium values of $G$ with increasing $w$ (Chang and Tsai 1991). At the same time, Carter (1992) found that as $w$ is increased, computed values of $G$ and $A$ more closely correspond to their 'reference' values. Moreover, upon increasing $w$, some changes in maps of topographic variables can be interpreted not as a decrease in the map accuracy but as generalization, that is, visualization of landform characteristics of other scales (Phillips 1988).

So, we believe that the accuracy of $G, A, k_{h}$ and $k_{v}$ derivation cannot be studied adequately by a comparison of calculated and 'reference' values. A radically different strategy should be used. It is obvious that the accuracy of data on $G, A, k_{h}$ and $k_{v}$ principally depends on:

- the accuracy of initial data, that is the DEM;

- precision of a calculation technique.

So, attention has to be focused on these two main factors of error generation. Thus, Felicisimo (1995) found that errors of $G$ increase with increasing root mean square error (RMSE) of a DEM. Brown and Bara (1994) and Giles and Franklin (1996) intimated that errors in calculations of partial derivatives of $z$ increase with the noise contained in the DEM. Skidmore (1989) and Hodgson (1995) compared accuracy of different methods for $G$ and $A$ derivation, that is calculation of $p$ and $q$ ( $\$ 4)$. Skidmore (1989) found that $p / q$ algorithms using data on six points of the 3 by 3 elevation submatrix $(\$ 2)$ are more accurate than a $p / q$ algorithm using data on four points of this submatrix. At the same time, Hodgson (1995) argued that a four-point algorithm is more accurate than six-point ones. Unfortunately, all these studies were also carried out with a comparison of calculated and 'reference' values of topographic variables.

It is clear that $G, A, k_{h}$ and $k_{v}$ are functions $F$ of measured variables $F=$ $\phi(x, y, \ldots, u)$ where $x, y, \ldots, u$ are measured arguments. In this case, measured arguments are $r, t, s, p$ and $q(\$ 4)$. Kuryakova (1996) proposed that the RMSE of $F$ $\left(m_{F}\right)$ would be appropriate to evaluate the accuracy of data on $G, A, k_{h}$ and $k_{v}$. To estimate $m_{F}$ the following formula can be applied (Gaidaev and Bolshakov 1969, p. 129):

$$
m_{F}=\sqrt{\left(\frac{\delta F}{\delta x}\right)_{0}^{2} m_{x}^{2}+\left(\frac{\delta F}{\delta y}\right)_{0}^{2} m_{y}^{2}+\ldots+\left(\frac{\delta F}{\delta u}\right)_{0}^{2} m_{u}^{2}}
$$

where $m_{x}, m_{y}, \ldots, m_{u}$ are RMSE of $x, y, \ldots, u$, correspondingly. In our opinion, this approach is best matched to the problem at hand.

Kuryakova (1996) produced equations for RMSE of $A$ and $G\left(m_{A}\right.$ and $m_{G}$, correspondingly). As measured arguments, she used partial derivatives of $z$ calculated by the method of Evans (1980) (\$2.1). Unfortunately, the development of the equation for $m_{G}$ was in error. Also Kuryakova (1996) did not justify using of the Evans method to calculate $r, t, s, p$ and $q$, although these derivatives can be estimated for other algorithms (Sharpnack and Akin 1969, Horn 1981, Papo and Gelbman 1984, Ritter 1987, Zevenbergen and Thorne 1987, Skidmore 1989, Moore et al. 1993, Shary 1995).

The objective of this study is to investigate the accuracy of data on $G, A, k_{h}$ and $k_{v}$. First, we determine the most precise method for calculation of $r, t, s, p$ and $q$ using a comparison of four often-used algorithms. Second, we produce formulae for $m_{G}, m_{A}$ and RMSE of $k_{h}$ and $k_{v}\left(m_{k_{h}}\right.$ and $m_{k_{v}}$ correspondingly), provided that $G, A$, 
$k_{h}$ and $k_{v}$ are evaluated with the most precise method for $r, t, s, p$ and $q$ calculation. Third, we demonstrate a practical implementation of formulae for $m_{G}, m_{A}, m_{k_{h}}$ and $m_{k_{r}}$.

\section{Four methods for calculation of $r, t, s, p$ and $q$}

Let us consider in details four often-used methods for calculation of $r, t, s, p$ and $q$, namely the methods of Evans (1980), Zevenbergen and Thorne (1987), Moore et al. (1993) and Shary (1995).

\subsection{The Evans method}

In the method of Evans (1980), the polynomial

$$
z=\frac{r x^{2}}{2}+\frac{t y^{2}}{2}+{ }_{s x y}+p x+q y+u
$$

is approximated by the least squares method to the 3 by 3 altitude submatrix. Points of the submatrix $\left(-w, w, z_{1}\right),\left(0, w, z_{2}\right),\left(w, w, z_{3}\right),\left(-w, 0, z_{4}\right),\left(0,0, z_{5}\right),\left(w, 0, z_{6}\right)$, $\left(-w,-w, z_{7}\right),\left(0,-w, z_{8}\right)$ and $\left(w,-w, z_{9}\right)$ are measured coordinates of the landsurface. As a result, we can estimate values of $r, t, s, p$ and $q$ at the point $\left(0,0, z_{5}\right)$ by the following formulae:

$$
\begin{gathered}
r=\frac{z_{1}+z_{3}+z_{4}+z_{6}+z_{7}+z_{9}-2\left(z_{2}+z_{5}+z_{8}\right)}{3 w^{2}}, \\
r=\frac{z_{1}+z_{2}+z_{3}+z_{7}+z_{8}+z_{9}-2\left(z_{4}+z_{5}+z_{6}\right)}{3 w^{2}}, \\
s=\frac{z_{3}+z_{7}-z_{1}-z_{9}}{4 w^{2}}, \\
p=\frac{z_{3}+z_{6}+z_{9}-z_{1}-z_{4}-z_{7}}{6 w}, \\
q=\frac{z_{1}+z_{2}+z_{3}-z_{7}-z_{8}-z_{9}}{6 w} .
\end{gathered}
$$

Moving the 3 by 3 submatrix along a regular DEM we can calculate values of $r, t$, $s, p$ and $q$ for all points of the DEM, excepting boundary points. Equations (3-7) were first published by Pennock et al. (1987). Sharpnack and Akin (1969) proposed expressions for $p$ and $q$ identical with equations (6 and 7).

The polynomial (equation (2)) is approximated to $z$ values of the 3 by 3 submatrix rather than passes exactly through these values. This leads to some smoothing of $z$ function within the 3 by 3 submatrix, that is, to local filtering of high-frequency noise resulted from small random errors in DEM compilation (Shary 1995). This low-pass filtering can provide more correct calculation of derivatives. This is because derivatives are very responsive to high-frequency components of a signal (Pratt 1978, Brown and Bara 1994, Giles and Franklin 1996). Excessive measurements are a further merit of the Evans method: nine values of $z$ are used to estimate six coefficients of the polynomial (equation (2)). This leads to improvements in the accuracy and tolerance of these computations (Bugaevsky, personal communication 1993). 


\subsection{The Zevenbergen and Thorne method}

In this method a Lagrange polynomial

$$
z=a x^{2} y^{2}+b x^{2} y+c x y^{2}+\frac{r x^{2}}{2}+\frac{t y^{2}}{2}+s x y+p x+q y+u
$$

passes exactly through all points of the 3 by 3 submatrix. Zevenbergen and Thorne (1987) anticipated that this condition may improve the calculation accuracy of partial derivatives. However, an opposite result can be produced due to the lack of excessive measurements, and availability of high-frequency noise of a DEM. Value of $s$ at the point $\left(0,0, z_{5}\right)$ of the 3 by 3 submatrix is estimated by equation (5), while values of $r, t, p$ and $q$ are evaluated by the following formulae:

$$
\begin{gathered}
r=\frac{z_{4}+{ }_{z_{6}}-2 z_{5}}{2 w^{2}}, \\
t=\frac{z_{2}+{ }_{z 8}-2 z_{5}}{2 w^{2}}, \\
p=\frac{z_{6}-z_{4}}{2 w}, \\
q=\frac{z_{2}-z_{8}}{2 w} .
\end{gathered}
$$

Ritter (1987) proposed expressions for $p$ and $q$ identical with equations (11 and 12).

\subsection{The Moore et al. method}

In the method of Moore et al. (1993), equations (5, 11, and 12) are used to calculate values of $s, p$ and $q$, respectively, at the point $(0,0, z 5)$ of the 3 by 3 submatrix, while values of $r$ and $t$ are calculated by the following formulae:

$$
\begin{aligned}
& r=\frac{z_{4}+z_{6}-2 z 5}{w^{2}}, \\
& t=\frac{z_{2}+z_{8}-2 z 5}{w^{2}} .
\end{aligned}
$$

\subsection{The Shary method}

Shary (1995) imposed the following condition: the polynomial (equation (2)) has to pass exactly through the point $\left(0,0, z_{5}\right)$ of the 3 by 3 submatrix, that is, $u=z 5$. With this method, values of $s, p$ and $q$ are calculated by equations (5-7), respectively, while values of $r$ and $t$ are computed by the following formulae:

$$
\begin{aligned}
& r=\frac{z_{1}+z_{3}+{ }_{z 7}+z_{29}+3\left(z_{4}+z_{6}\right)-2\left(z_{2}+3 z_{5}+z_{8}\right)}{5 w^{2}}, \\
& t=\frac{z_{1}+{ }_{z 3}+{ }_{z 7}+{ }_{z 9}+3\left(z_{2}+z_{8}\right)-2\left(z_{4}+3 z_{5}+z_{6}\right)}{5 w^{2}} .
\end{aligned}
$$




\section{Test for the precision of methods for calculation of $r, t, s, p$ and $q$}

$r, t, s, p$ and $q$ (equations (3-7) (9-16)) are functions $F$ of measured variables. Measured arguments are $z i, i=1, \ldots, 9$. We carry out the test for the precision of methods for calculation of partial derivatives of $z$ with the criterion of $m_{F}$ (equation (1)). It should be stressed that we examine the fundamental error in the algorithms rather than an error associated with how well polynomials are used within those methods (equations ( 2 and 8 )) to model the real elevation distribution. This problem is rather complicated (e.g., Lobanov and Zhurkin 1980, Carter 1988) and is outside the scope of the present study.

Let us produce formulae of RMSE of $r, t, s, p$ and $q\left(m_{r}, m_{t}, m_{s}, m_{p}\right.$ and $m_{q}$, respectively) for the methods described (\$2). In particular, for the Evans method

$$
m_{r}=\sqrt{\left(\frac{\delta_{r}}{\delta_{z_{1}}}\right)_{0}^{2} m_{z_{1}}^{2}+\left(\frac{\delta_{r}}{\delta_{z_{2}}}\right)_{0}^{2} m_{z_{2}}^{2}+\left(\frac{\delta_{r}}{\delta_{z_{3}}}\right)_{0}^{2} m_{z_{3}}^{2}+\ldots+\left(\frac{\delta_{r}}{\delta_{z_{9}}}\right)_{0}^{2} m_{z_{9}}^{2}}
$$

where $m_{z_{1}}, m_{z_{2}}, m_{z_{3}}, \ldots, m_{z_{\mathrm{g}}}$ are RMSE of $z_{1}, z_{2}, z_{3}, \ldots, z_{9}$. In the strict sense, $m_{z_{i}}=$ $\phi(x, y), x$ and $y$ are planimetric coordinates. $m_{z_{i}}$ depends on geomorphic conditions, methods of compilation and interpolation of a DEM (Hunter and Goodchild 1995). Ackermann (1978) and Li (1994) proposed formulae for $m_{z_{i}}$ estimation. According to these expressions, $m_{z_{i}}$ is a function of $G$. However, it is not profitable to use formulae of this kind because the accuracy of calculations of $G$ depends on $m_{z_{i}}$ too (Felicisimo 1995).

At the same time, for a DEM produced by digitizing contours

$$
m_{z_{i}}=\text { const }=B h
$$

where $B=0 \cdot 16-0 \cdot 33, h$ is a contour interval ( $\mathrm{Li} 1994)$. The factor $B$ depends on geomorphic conditions, and the extent to which additional feature-specific data (i.e., peaks, pits, watersheds, thalvegs) are incorporated into the DEM. So, let us consider $m_{z_{1}}=m_{z_{2}}=\ldots=m_{z_{9}}=m_{z}$. Substituting $m_{z}$ into equation (17) gives:

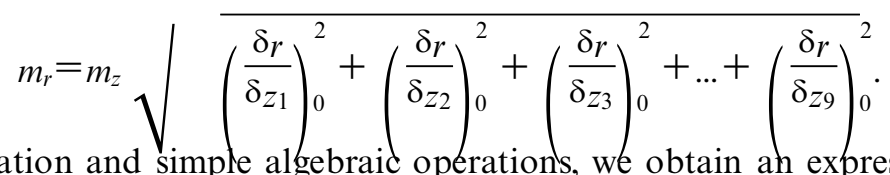

On differentiation and simple algebraic operations, we obtain ah expression of $m_{r}$ for the Evans method (table 1). Rearrangements can be dropped. In a similar manner,

Table 1. $m_{r}, m_{t}, m_{s}, m_{p}$ and $m_{q}$ for different methods.

\begin{tabular}{lccc}
\hline Method & $m_{r}=m_{t}$ & $m_{s}$ & $m_{p}=m_{q}$ \\
\hline The Evans method & $\frac{1 \cdot 41 m_{z}}{w^{2}}$ & $\frac{0 \cdot 5 m_{z}}{w^{2}}$ & $\frac{0 \cdot 41 m_{z}}{w}$ \\
The Zevenbergen and Thorne method & $\frac{1 \cdot 22 m_{z}}{w^{2}}$ & $\frac{0 \cdot 5 m_{z}}{w^{2}}$ & $\frac{0 \cdot 71 m_{z}}{w}$ \\
The Moore et al. method & $\frac{2 \cdot 45 m_{z}}{w^{2}}$ & $\frac{0 \cdot 5 m_{z}}{w^{2}}$ & $\frac{0 \cdot 71 m_{z}}{w}$ \\
The Shary method & $\frac{1 \cdot 62 m_{z}}{w^{2}}$ & $\frac{0 \cdot 5 m_{z}}{w^{2}}$ & $\frac{0 \cdot 41 m_{z}}{w}$ \\
\hline
\end{tabular}


we derive the required expressions of $m_{r}, m_{t}, m_{s}, m_{p}$ and $m_{q}$ for all methods tested (table 1).

$m_{r}$ equals $m_{t}$, and $m_{p}$ equals $m_{q}$ for all methods (table 1). Values of RMSE obtained are in direct proportion to $m_{z} . m_{p}$ and $m_{q}$ are in inverse proportion to $w$, while $m_{r}, m_{t}$ and $m_{s}$ are in inverse proportion to $w^{2}$ (table 1). So, second partial derivatives are more responsive than first partial derivatives to changes in $w$. It should be emphasized that with low values of $w, m_{r}, m_{t}$ and $m_{s}$ values far exceed $m_{p}$ and $m_{q}$ ones, while with high values of $w, m_{p}$ and $m_{q}$ values far exceed $m_{r}, m_{t}$ and $m_{s}$ ones (table 2).

Equation (5) is used to estimate $s$ values by all methods tested ( $\$ 2)$. So, the precision of the methods is dictated by $m_{r}, m_{t}, m_{p}$ and $m_{q}$. Comparative analysis of the formulae obtained (table 1) demonstrated that the Moore et al. method is marked by the highest values of $m_{r}$ and $m_{t}$. The Zevenbergen and Thorne method and the Moore et al. method are equal in $m_{p}$ and $m_{q}$. Also, the Evans method and the Shary method are equal in $m_{p}$ and $m_{q}$. However, $r$ and $t$ calculation is more accurate with the Evans method than with the Shary method. $r$ and $t$ are further precisely evaluated by the Zevenbergen and Thorne method. At the same time, $p$ and $q$ calculation is more accurate with the Evans method than with the Zevenbergen and Thorne method. Considering these facts and drawbacks of the Zevenbergen and Thorne method (\$2.2), we can conclude that the Evans method is the most precise algorithm for estimation of $r, t, s, p$ and $q$. Note, that although the Evans method is least affected by elevation errors, that does not mean that the Evans polynomial (equation (2)) best represent elevation reality.

It should be noticed that our results (table 1) conform with conclusions of Skidmore (1989) rather than with results of Hodgson (1995): $p$ and $q$ derivation is more accurate with the Evans and the Shary methods (six-point algorithms) than with the Zevenbergen and Thorne and the Moore et al. methods (four-point algorithms).

\section{Development of $m_{G}, m_{A}, m_{k_{h}}$ and $m_{k_{v}}$ formulae}

$G, A, k_{h}$ and $k_{v}$ can be calculated with the following formulae (Shary 1991):

$$
\begin{gathered}
G=\operatorname{arctg}\left(\sqrt{p^{2}+q^{2}}\right), \\
A=\operatorname{arctg}\left(\begin{array}{c}
q \\
p
\end{array}\right), \\
k_{h}=-\frac{q^{2} r-2 p q s+p^{2} t}{\left(p^{2}+q^{2}\right) \sqrt{1+p^{2}+q^{2}}}, \\
k_{v}=-\frac{p^{2} r+2 p q s^{2}+q^{2} t}{\left(p^{2}+q^{2}\right) \sqrt{\left(1+p^{2}+q^{2}\right)^{3}}} .
\end{gathered}
$$

Table 2. Relations between $w$ and $m_{r}, m_{t}, m_{s}, m_{p}$ and $m_{q}$ for the Evans method, $m_{z}=1 \mathrm{~m}$.

\begin{tabular}{lcllll}
\hline$w, m$ & $0 \cdot 1$ & 1 & 10 & 100 & 1000 \\
\hline$m_{r}=m_{t}$ & 141 & $1 \cdot 41$ & $0 \cdot 0141$ & $0 \cdot 000141$ & $0 \cdot 00000141$ \\
$m_{s}=m_{p}$ & 50 & $0 \cdot 5$ & $0 \cdot 005$ & $0 \cdot 00005$ & $0 \cdot 0000005$ \\
$m_{p}$ & $4 \cdot 1$ & $0 \cdot 41$ & $0 \cdot 041$ & $0 \cdot 0041$ & $0 \cdot 00041$ \\
\hline
\end{tabular}


Equations (20-23) should be tested to determine $m_{G}, m_{A}, m_{k_{h}}$ and $m_{k_{v}}$ although some simplified expressions of $G, A, k_{h}$ and $k_{v}$ are not uncommon in the literature (e.g., Papo and Gelbman 1984, Skidmore 1989). However, equations (20-23) most closely correspond to the physical and mathematical theory of surface in gravity (Shary 1991, 1995).

Let us derive expressions for $m_{G}, m_{A}, m_{k_{h}}$ and $m_{k_{v}}$ with equation (1). As measured arguments, we use $r, t, s, p$ and $q$ calculated by the Evans method (equations 3-7). This is because the Evans method is the most precise technique for evaluation of these partial derivatives $(\S 3) . m_{G}, m_{A}, m_{k_{h}}$ and $m_{k_{v}}$ are developed in a manner like $m_{r}, m_{t}, m_{s}, m_{p}$ and $m_{q}$ (table 1) were derived $(\$ 3)$. Through differentiation, simple algebraic operations and substitutions we can obtain the following expressions:

$$
\begin{aligned}
& m_{G}=\sqrt{\left(\frac{\delta G}{\delta p}\right)_{0}^{2} m_{p}^{2}+\left(\frac{\delta G}{\delta q}\right)_{0}^{2} m_{q}^{2}}=\ldots=\frac{0 \cdot 41 m_{z}}{w\left(1+p^{2}+q^{2}\right)},
\end{aligned}
$$

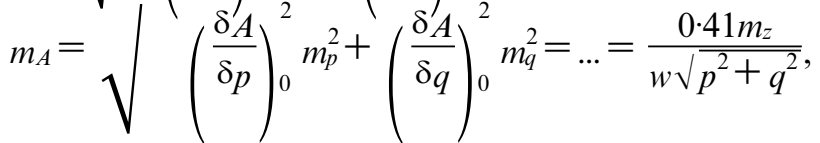

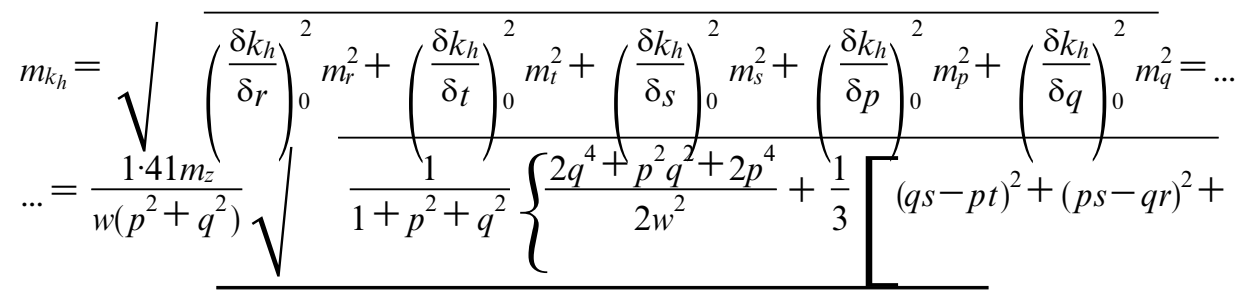

$$
\begin{aligned}
& \left.\left.+\frac{\left(q^{2} r-2 p q s+p^{2} t\right)\left(p^{2}+q^{2}\right)}{4\left(1+p^{2}+q^{2}\right)^{2}}-\frac{\left(q^{2} r-2 p q s+p^{2} t\right)^{2}}{p^{2}+q^{2}}\right]\right\} \text {, } \\
& \ldots=\frac{1 \cdot 41 m_{z}}{w\left(p^{2}+q^{2}\right)} \sqrt{\frac{\left(\frac{\delta k_{v}}{\delta r}\right)_{0}^{2} m_{r}^{2}+\left(\frac{\delta k_{v}}{\delta t}\right)_{0}^{2} m_{t}^{2}+\left(\frac{\delta k_{v}}{\delta s}\right)_{0}^{2} m_{s}^{2}+\left(\frac{\delta k_{v}}{\delta p}\right)_{0}^{2} m_{p}^{2}+\left(\frac{\delta k_{v}}{\delta q}\right)_{0}^{2} m_{q}^{2}}{\frac{1}{\left(1+p^{2}+q^{2}\right)^{3}}\left\{\frac{2 q^{4}+p^{2} q^{2}+2 p^{4}}{2 w^{2}}+\frac{1}{3}\left[(q s+p r)^{2}+(p s+q t)^{2}+\right.\right.}} \\
& \left.\left.+\frac{9\left(p^{2} r+2 p q s+q^{2} t\right)\left(p^{2}+q^{2}\right)}{4\left(1+p^{2}+q^{2}\right)^{2}}-\frac{\left(p^{2} r+2 p q s+q^{2} t\right)^{2}}{p^{2}+q^{2}}\right]\right\}
\end{aligned}
$$

We can determine some general properties of $m_{G}, m_{A}, m_{k_{h}}$ and $m_{v}$ functions by a simple analysis of equations (24-27). First, it is clear that values of these RMSE are in direct proportion to $m_{z}$, and in inverse proportion to $w$ (equations 24 and 25) or $w^{2}$ (equations 26 and 27). So, $m_{G}, m_{A}, m_{k_{h}}$ and $m_{k_{v}}$ increase with decreasing $w$. This property of equations (24-27) correlates well with some findings of the investigation performed by Carter (1992) $(\S 1)$. It is obvious that $k_{h}$ and $k_{v}$ are more responsive than $G$ and $A$ to changes in $w$. Second, the factor of $G$, namely, $\left(p^{2}+q^{2}\right)$ is predominantly in denominators of equations (24-27). So, $m_{G}, m_{A}, m_{k_{h}}$ and $m_{k_{v}}$ can become large with decreasing $G$, for instance, in flat areas. This property of equations (24-27) also conforms with some results of studies carried out by Chang and Tsai (1991) and Carter (1992) $(\S 1)$. 
The following question arises: How we can use equations (24-27) in actual practice? Mapping is a convenient and pictorial strategy to visualize propagation of errors in spatial modelling (Heuvelink et al. 1989, Kraus 1994, Hunter and Goodchild 1995). So, equations (24-27) would be appropriate for use in mapping of $m_{G}, m_{A}$, $m_{k_{h}}$ and $m_{k_{r}}$.

\section{Mapping of $\boldsymbol{m}_{G}, \boldsymbol{m}_{A}, \boldsymbol{m}_{k_{h}}$ and $\boldsymbol{m}_{k_{v}}$}

\subsection{Materials and methods}

We used a DEM of a territory adjoining the town of L'gov (Kursk Region, Russia) (figure 1) to demonstrate the possibilities of mapping of $m_{G}, m_{A}, m_{k_{h}}$ and $m_{k_{v}}$. The study area measures $68 \mathrm{~km}$ by $48 \mathrm{~km}$. The irregular DEM of the study site was compiled by digitizing contours and specific features of relief (i.e., some peaks, watersheds and thalvegs) from a 1: 200000 topographic map (GENERAL HEADQUARTERS 1981). The irregular DEM includes about 47000 points. The regular DEM (figure 1) was generated by the irregular DEM interpolation using the weighted average method (Schut 1976). We applied a matrix step of $180 \mathrm{~m}$. We calculated digital models of $G$ (figure 2(a)), $A$ (figure 2(c)), $k_{h}$ (figure 2(e)) and $k_{v}$ (figure 2(g)) by the method of Evans (1980) with $w$ of $1500 \mathrm{~m}$.

Contour interval is $20 \mathrm{~m}$ within the topographic map used. We set the factor $B=$ $0 \cdot 25$, since some specific features of relief were digitized. According to equation (18), we applied $m_{z}=5 \mathrm{~m}$ in calculation and mapping of $m_{G}$ (figure 2(b)), $m_{A}$ (figure 2(d)), $m_{k_{h}}$ (figure 2(f)) and $m_{k_{v}}$ (figure 2(h)).

Visualization of DTMs marked by low resolution, as a rule, leads to production of poorly readable maps (e.g., Papo and Gelbman 1984). To improve visual perception we used a smooth interpolation (Schut 1976) of $G, m_{G}, k_{h}, m_{k_{h}}, k_{v}$ and $m_{k_{v}}$ in mapping (figures $2(a, b, e-h)$ ). $A$ values (figure 2(c)) were not smoothed due to the particular feature of $A$ (see its definition in $\S 1$ ). Interpolation of $A$ can lead to some artefacts. For example, assume that two neighbouring points I and II are marked

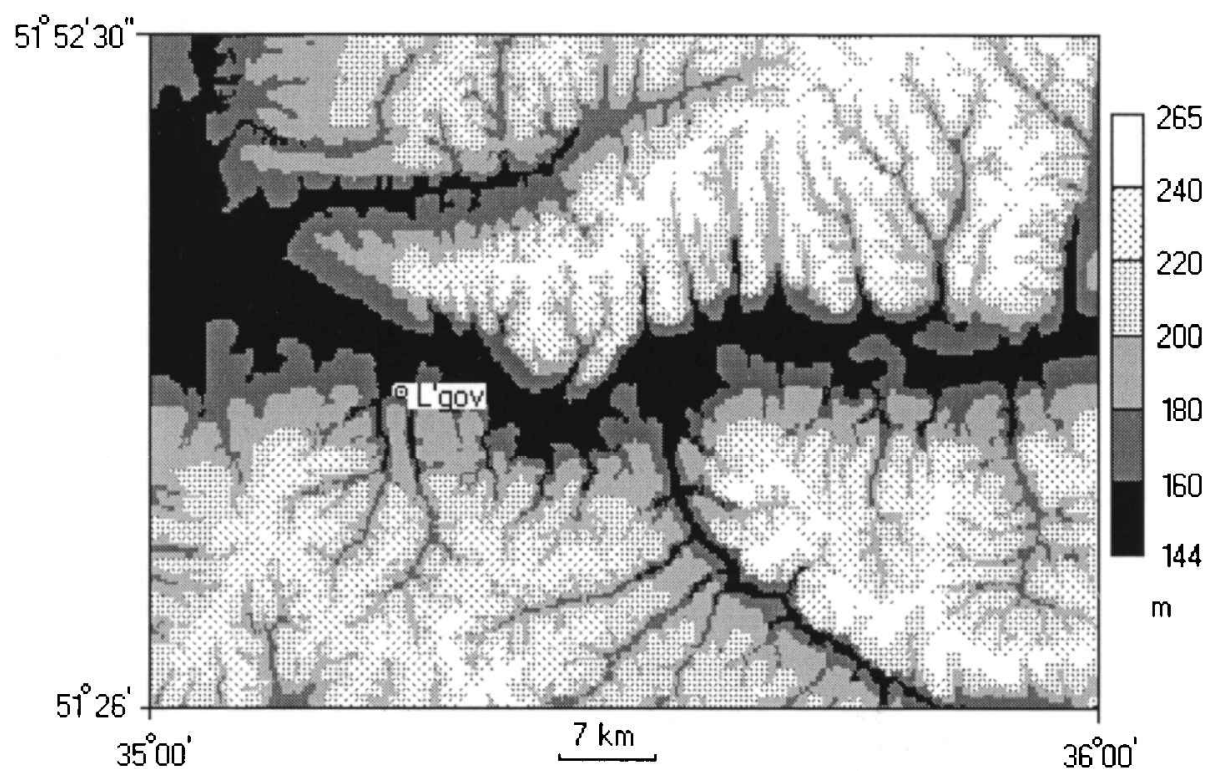

Figure 1. Elevation map of the part of the Kursk Region (Russia). 







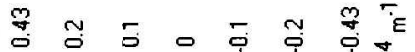



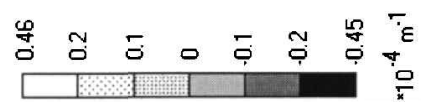



$=$

in

造

过 m ญै

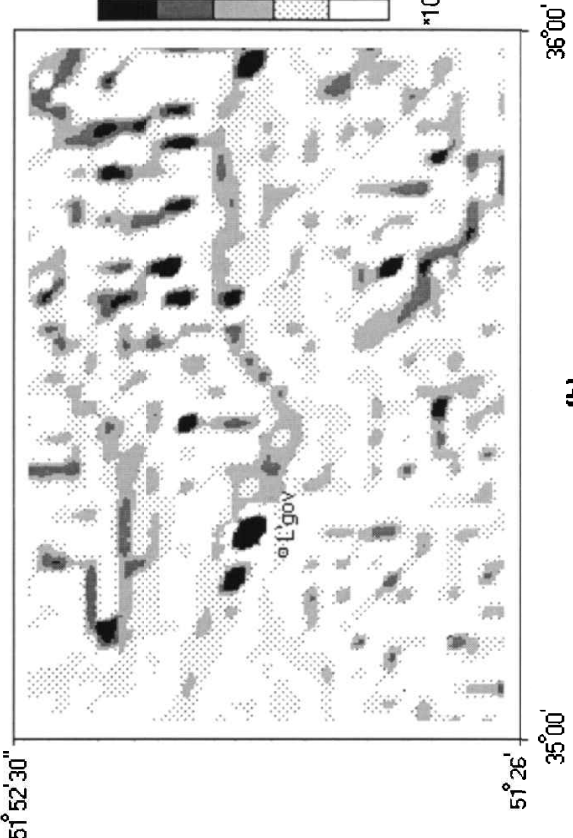

m

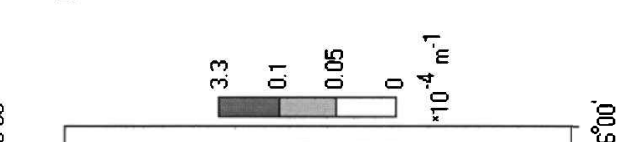

通

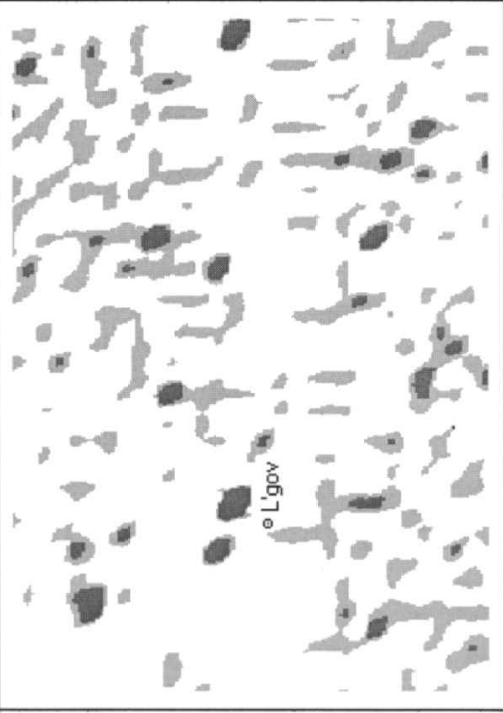

=е $\left.\frac{\xi}{m}\right]$

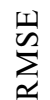

Ð

过

co

氧 कू

पे

넹

$\sum \frac{\omega}{0}$

$\equiv \quad \stackrel{\sim}{2}$

武

is

क力

记

$\sum_{\simeq}^{\infty} \frac{0}{0}$

$\because \frac{7}{0}$

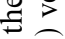

Dै

范

胥莡

.

플

천 글

कू

융

핀됴

은

पू $\simeq$

纪

है

$\Xi$

(ิ)

党

홍

का

$\simeq$

空

可

ปี

F

ते

$\stackrel{0}{\leftrightarrows}$ 
Table 3. Pairwise coefficients of correlations between local topographic variables and their RMSE (in brackets are significance levels), for the part of the Kursk Region.

\begin{tabular}{ccccrr}
\hline & & $G$ & \multicolumn{1}{c}{$A$} & \multicolumn{1}{c}{$k_{h}$} & \multicolumn{1}{c}{$k_{v}$} \\
\hline$m_{G}$ & $-0.03(0.34)$ & $-0.95(0.00)$ & $-0.01(0.66)$ & $-0.16(0.00)$ & $-0.09(0.00)$ \\
$m_{A}$ & $-0.07(0.01)$ & $-0.15(0.00)$ & $-0.05(0.07)$ & $0.01(0.61)$ & $-0.03(0.29)$ \\
$m_{k h}$ & $-0.01(0.69)$ & $-0.16(0.00)$ & $-0.06(0.04)$ & $-0.18(0.00)$ & $-0.08(0.00)$ \\
$m_{k v}$ & $-0.03(0.24)$ & $-0.09(0.00)$ & $-0.04(0.15)$ & $-0.09(0.00)$ & $-0.47(0.00)$ \\
\hline
\end{tabular}

by $A$ values of 10 and 385 degrees, correspondingly. Upon interpolating, $A$ takes values 10-385 degrees at points located between points I and II. So, we obtain the artefact, that is, alternating small zones of north-east, east, south-east, south, south-west, west and north-west aspects. Also, we did not interpolate $m_{A}$ values (figure 2(d)) for convenient comparison of A and $m_{A}$ maps (figures 2(c, d)).

To estimate quantitatively spatial relations between topographic variables and RMSE of their calculation, we carried out a linear correlative analysis between $m_{G}$, $m_{A}, m_{k_{h}}, m_{k_{v}}$ and $z, G, A, k_{h}, k_{v}$. We used a 1364-point sample. The sample step was $1500 \mathrm{~m}$. The results of correlative analysis are shown in table 3.

We applied the software LANDLORD 2.1 (Florinsky et al. 1995) for the irregular DEM interpolation, calculation and mapping of topographical variables and their RMSE (figures 1,2). Correlative analysis was carried out by the software STATGRAPHICS 2.6 .

\subsection{Results and discussion}

Maps of $m_{G}, m_{A}, m_{k_{h}}$ and $m_{k_{v}}$ obtained (figures $2(b, d, f, h)$ ) clearly demonstrate the spatial distribution of these RMSE within the study site. Analysis of the maps (figure 2) and correlation coefficients (table 3 ) allowed us to determine some regularities for the spatial distribution of $m_{G}, m_{A}, m_{k_{h}}$ and $m_{k_{v}}$. An inverse dependence of $m_{G}$ on $G$ is the most conspicuous association (table 3, figures $2(a, b)$ ). This result fits well with the conclusion about $G$ errors obtained by Carter (1992) $(\$ 1)$. Maximum values of $m_{A}$ are also observed within flat areas (figures $2(a, d)$ table 3 ). In some cases, $m_{A}$ ranges up to tens of degrees there (figures $2(a, d)$ ). This result correlates with the inferences about $A$ errors obtained by Chang and Tsai (1991) and Carter (1992) $(\S 1)$. However, $m_{G}$ and $m_{A}$ values are generally negligible (figures $2(b, d)$ ). High values of $m_{k_{h}}$ and $m_{k_{v}}$ are typical for flat areas too (figures $2(a, f, h)$ ). In addition, there is a strong negative correlation between $m_{k_{v}}$ and $k_{v}$, and small inverse dependencies of $m_{k_{h}}$ on $k_{h}$ and $G$ (table 3). It must be emphasized that values of $m_{k_{h}}$ and $m_{k_{v}}$ can be in excess of maximum values of $k_{h}$ and $k_{v}$, respectively, within some flat areas (figures $2(a, e-h)$ ). $A$ does not effect propagation of $m_{G}, m_{A}, m_{k_{h}}$ and $m_{k_{v}}$ (table 3 ). Notice that the results of correlative analysis (table 3 ) describe trends of relations between topographic variables and their RMSE. It is likely that these relations can depend on geomorphic conditions also.

The regularities of spatial distribution of $m_{G}, m_{A}, m_{k_{h}}$ and $m_{k_{v}}$ should be taken into account in landscape investigations with DTMs. Researchers have to treat data on local topographic variables (notably $k_{h}$ and $k_{v}$ ) with criticism, especially in studies of plain terrains. Data on $m_{G}, m_{A}, m_{k_{h}}$ and $m_{k_{v}}$ can be used:

- to account for a spatial distribution of $m_{F}$ in analysis and interpretation of $F$ (Carter 1992); 
- to refine a DEM within areas marked by high values of $m_{F}$, and then to re-calculate $F$ within these areas (Hunter and Goodchild 1995);

- to correct errors of $F$ calculated by some methods including combined processing of data on $m_{F}$ and $F$ (Heuvelink et al. 1989).

DEMs are also applied to calculate non-local topographic variables, such as specific catchment area, and to reveal thalveg and watershed networks (Moore et al. 1991, Shary et al. 1991). Precision of these techniques has not been adequately explored (Skidmore 1990, Lee et al. 1992). However, these problems are outside the scope of the present study.

\section{Conclusions}

We studied the accuracy of data on $G, A, k_{h}$ and $k_{v}$. First, we carried out the test for the precision of four methods for calculation of $r, t, s, p$ and $q$. We found that the Evans method is the most precision algorithm of this kind. Second, we produced formulae for $m_{G}, m_{A}, m_{k_{h}}$ and $m_{k_{n}}$ provided that $G, A, k_{h}$ and $k_{v}$ are evaluated with the Evans method. Third, we demonstrated that mapping is the most convenient and pictorial way for the practical implementation of the formulae derived. The DEM of the part of the Kursk Region (Russia) was used as an example. We found that high values of $m_{G}, m_{A}, m_{k_{h}}$ and $m_{k_{v}}$ are typical for flat areas. Results obtained can be used to improve landscape investigations with DTMs.

\section{Acknowledgements}

The author is grateful to Dr. G. A. Kuryakova, Professor Yu. I. Markuze (Moscow State University of Geodesy and Cartography, Moscow, Russia) and Dr. P. A. Shary (Institute of Soil Science and Photosynthesis, Russian Academy of Sciences, Pushchino, Russia) for fruitful discussions, as well as Mr. P. V. Kozlov (ZAO "IC Protek", Moscow, Russia) for technical assistance. This work was carried out with partial support from NATO grant ENVIR.CRG 950218.

\section{References}

ACKERMANN, F., 1978, Experimental investigation into the accuracy of contouring from DTM. Photogrammetric Engineering and Remote Sensing, 44, 1537-1548.

Ames, W. F., 1977, Numerical Methods for Partial Differential Equations (New York: Academic Press).

Bolstad, P. V., and Stowe, T., 1994, An evaluation of DEM accuracy: Elevation, slope and aspect. Photogrammetric Engineering and Remote Sensing, 60, 1327-1332.

Brown, D. G., and BARA, T. J., 1994, Recognition and reduction of systematic error in elevation and derivative surfaces from 7-5-minute DEMs. Photogrammetric Engineering and Remote Sensing, 60, 189-194.

Burrough, P. A., 1986, Principles of Geographical Information Systems for Land Resources Assessment (Oxford: Clarendon Press).

Carter, J. R., 1988, Digital representations of topographic surfaces. Photogrammetric Engineering and Remote Sensing, 54, 1577-1580.

CARTER, J. R., 1992, The effect of data precision on the calculation of slope and aspect using gridded DEMs. Cartographica, 29, 22-34.

Chang, K.-T., and Tsai, B.-W., 1991, The effect of DEM resolution on slope and aspect mapping. Cartography and Geographical Information Systems, 18, 69-77.

Davis, F. W., and DoziER, J., 1990, Information analysis of a spatial database for ecological land classification. Photogrammetric Engineering and Remote Sensing, 56, 605-613.

Doyle, F. J., 1978, Digital terrain models: An overview. Photogrammetric Engineering and Remote Sensing, 44, 1481-1485.

Evans, I. S., 1980, An integrated system of terrain analysis and slope mapping. Zeitschrift für Geomorphologie, Suppl. Bd. 36, 274-295. 
Felicisimo, A. M., 1994a, Modelos Digitales del Terreno. Introducción y Aplicaciones en las Ciencias Ambientales (Oviedo: Pentalfa Ediciones).

Felicisimo, A. M., 1994b, Parametric statistical method for error detection in digital elevation models. ISPRS Journal of Photogrammetry and Remote Sensing, 49, 29-33.

Felicisimo, A. M., 1995, Error propagation analysis in slope estimation by means of digital elevation models. In Cartography Crossing Borders: Proceedings 1 of the 17th International Cartographic Conference and 10th General Assembly of International Cartographic Association (Barcelona: Institut Cartografic de Catalunya), pp. 94-98.

FLORINSKY, I. V., 1995, International experience of using digital elevation models in automated analysis of remotely sensed data. Geodesiya i Cartographiya, 12, 33-38 (in Russian).

Florinsky, I. V., Grokhlina, T. I., and Mikhailova, N. L., 1995, LANDlord 2.0: the software for analysis and mapping of geometrical characteristics of relief. Geodesiya $i$ Cartographiya, 5, 46-51 (in Russian).

Frederiksen, P., 1981, Terrain analysis and accuracy prediction by means of the Fourier transformation. Photogrammetria, 36, 145-157.

Gaidaev, P. A., and BolshaKov, V. D., 1969, Theory of Mathematical Processing of Geodetic Measurements (Moscow: Nedra) (in Russian).

General Headquarters, 1981, T opographic Map, scale 1:200,000. Page 13-36-06 (M-36VI ), L'gov. (Moscow: General Headquarters) (in Russian).

Giles, P. T., and Franklin, S. E., 1996, Comparison of derivative topographic surfaces of a DEM generated from stereoscopic SPOT images with field measurements. Photogrammetric Engineering and Remote Sensing, 62, 1165-1171.

Hannah, M. J., 1981, Error detection and correction in digital terrain models. Photogrammetric Engineering and Remote Sensing, 47, 63-69.

Heuvelink, G. B. M., Burrough, P. A., and Stein, A., 1989, Propagation of errors in spatial modelling with GIS. International Journal of Geographical Information Systems, 3, 303-322.

Hodgson, M. E., 1995, What cell size does the computed slope/aspect angle represent? Photogrammetric Engineering and Remote Sensing, 61, 513-517.

Horn, B. K. P., 1981, Hill shading and the reflectance map. Proceedings of the Institute of Electrical and Electronics Engineers, 69, 14-47.

Hunter, G. J., and GoodchiLd, M. F., 1995, Dealing with error in spatial databases: a simple case study. Photogrammetric Engineering and Remote Sensing, 61, 529-537.

Kraus, K., 1994, Visualization of the quality of surfaces and their derivatives. Photogrammetric Engineering and Remote Sensing, 60, 457-462.

Kumler, M. P., 1994, An intensive comparison of triangulated irregular networks (TINs) and digital elevation models (DEMs). Cartographica, 31, 1-99.

Kuryakova, G. A., 1996, Strategy for Investigation and Preparation of Initial Data for Biogeocoenosis Mapping with Digital Terrain Models: Abstract of Ph.D. Thesis (Moscow: Moscow State University of Geodesy and Cartography) (in Russian).

LeE, J., SNYDER, P. K., and Fisher, P. F., 1992, Modeling the effect of data errors on feature extraction from digital elevation models. Photogrammetric Engineering and Remote Sensing, 58, 1461-1467.

LI, Z., 1994, A comparative study of the accuracy of digital terrain models (DTMs) based on various data models. ISPRS Journal of Photogrammetry and Remote Sensing, 49, 2-11.

Lobanov, A. N., and Zhurkin, I. G., 1980, Automation of Photogrammetric Processes. (Moscow: Nedra) (in Russian).

Miller, C. L., and Leflamme, R. A., 1958, The digital terrain model - Theory and application. Photogrammetric Engineering, 24, 433-442.

Moore, I. D., Gessler, P. E., Nielsen, G. A., and Peterson, G. A., 1993, Soil attribute prediction using terrain analysis. Soil Science Society of America Journal, 57, 443-452.

Moore, I. D., Grayson, R. B., and Ladson, A. R., 1991, Digital terrain modelling: A review of hydrological, geomorphological and biological applications. Hydrological Processes, 5, 3-30.

Papo, H. B., and Gelbman, E., 1984, Digital terrain models for slopes and curvatures. Photogrammetric Engineering and Remote Sensing, 50, 695-701.

Pennock, D. J., Zebarth, B. J., and De Jong, E., 1987, Landform classification and soil distribution in hummocky terrain, Saskatchewan, Canada. Geoderma, 40, 297-315. 
Phillips, J. D., 1988, The role of spatial scale in geomorphic systems. Geographical Analysis, 20, 308-317.

Pratt, W. K., 1978, Digital Image Processing (New York: John Wiley and Sons).

RitTer, P., 1987, A vector-based slope and aspect generation algorithm. Photogrammetric Engineering and Remote Sensing, 53, 1109-1111.

Sasowsky, K. C., Petersen, G. W., and Evans, B.M., 1992, Accuracy of SPOT digital elevation model and derivatives: Utility for Alaska's North Slope. Photogrammetric Engineering and Remote Sensing, 58, 815-824.

Schut, G. H., 1976, Review of interpolation methods for digital terrain models. The Canadian Surveyor, 30, 389-412.

Sharpnack, D. A., and AKIn, G., 1969, An algorithm for computing slope and aspect from elevations. Photogrammetric Engineering and Remote Sensing, 35, 247-248.

Shary, P. A., 1991, The second derivative topographic method. In The Geometry of the Earth Surface Structures, edited by I.N. Stepanov (Pushchino: Pushchino Research Centre Press), pp. 30-60 (in Russian).

Shary, P. A., 1995, Land surface in gravity points classification by complete system of curvatures. Mathematical Geology, 27, 373-390.

Shary, P. A., Kuryakova, G. A., and Florinsky, I. V., 1991, On the international experience of topographic methods employment in landscape researches (the concise review). In The Geometry of the Earth Surface Structures, edited by I.N. Stepanov (Pushchino: Pushchino Research Centre Press), pp. 15-29 (in Russian).

Skidmore, A. K., 1989, A comparison of techniques for calculation gradient and aspect from a gridded digital elevation model. International Journal of Geographical Information Systems, 3, 323-334.

Skidmore, A. K., 1990, Terrain position as mapped from a gridded digital elevation model. International Journal of Geographical Information Systems, 4, 33-49.

Zevenbergen, L. W., and Thorne, C. R., 1987, Quantitative analysis of land surface topography. Earth Surface Processes and Landforms, 12, 47-56. 
